\title{
Lung cancer screening: assessment of health literacy and readability of online educational resources
}

\author{
Kevin Haas ${ }^{1 *}$ (D) Christie Brillante ${ }^{1}$, Lisa Sharp ${ }^{2}$, Ahmed K. Elzokaky ${ }^{1}$, Mary Pasquinelli ${ }^{1}$, Lawrence Feldman ${ }^{3}$, \\ Kevin L. Kovitz ${ }^{1}$ and Min Joo ${ }^{1}$
}

\begin{abstract}
Background: Lung cancer screening can reduce mortality but can be a complex, multi-step process. Poor health literacy is associated with unfavorable outcomes and decreased use of preventative services, so it is important to address barriers to care through efficient and practical education. The readability of lung cancer screening materials for patients is unknown and may not be at the recommended 6th grade reading level set by the American Medical Association. Our goals were to: (1) measure the health literacy of a lung cancer screening population from an urban academic medical center, and (2) examine the readability of online educational materials for lung cancer screening.

Methods: We performed a retrospective cross sectional study at a single urban academic center. Health literacy was assessed using three validated screening questions. To assess the readability of educational materials, we performed a Google search using the phrase, "What is lung cancer screening?" and the Flesch-Kincaid Grade Level (FKGL) formula was used to estimate the grade level required to understand the text.

Results: There were 404 patients who underwent lung cancer screening during the study period. The prevalence of inadequate/marginal health literacy was 26.7-38.0\%. Fifty websites were reviewed and four were excluded from analysis because they were intended for medical providers. The mean FKGL for the 46 websites combined was $10.6 \pm 2.2$.

Conclusions: Low health literacy was common and is likely a barrier to appropriate education for lung cancer screening. The current online educational materials regarding lung cancer screening are written above the recommended reading level set by the American Medical Association.
\end{abstract}

Keywords: Health literacy, Lung cancer screening, Patient education

\section{Background}

Lung cancer is the leading cause of cancer related deaths in the United States with a worse prognosis among the underserved and ethnic minority populations [1-3]. Early diagnosis is associated with improved mortality, yet more than $50 \%$ of lung cancer is diagnosed at an advanced stage $[4,5]$. The National Lung Cancer Screening Trial (NLST) demonstrated a $20 \%$ mortality reduction from lung cancer using annual computed tomography to screen high risk patients [3]. Like much of cancer research however, the sample was $90 \%$ white [6-11]. Thus

\footnotetext{
* Correspondence: kphaas@uic.edu

${ }^{1}$ University of Illinois at Chicago, 840 S. Wood St., CSB 915, MC 719, Chicago, IL 60612, USA

Full list of author information is available at the end of the article
}

little is known about the impact of sociodemographic factors such as race, ethnicity, income, education, and health literacy on lung cancer screening (LCS) outcomes. This is important since a low socioeconomic status (SES) limits a patients' ability to follow cancer screening recommendations and often results in finding more advanced disease $[3,12]$.

Primary care physicians (PCP) and pulmonologists play a vital role in LCS and comprise the majority of ordering physicians [13]. Counseling for lung cancer screening should involve a shared decision making discussion between the ordering physician and their patient [14]. The lung cancer screening process can be complex and involve several diagnostic pathways, referrals, and procedures the patient will need to consider if an 
abnormality is found. The discussion should include the risks and benefits of screening, appropriate follow up, implications of false positive findings, radiation exposure, and smoking cessation [14]. The ordering physician is ultimately responsible for patient education about lung cancer screening. Time constraints and limited resources are known barriers to LCS making patient education in a busy clinic environment a challenge [13, 1518]. This can lead to many patients using the internet for their health information [19]. Improved educational modalities, especially for low literacy patients, are needed to improve the screening process.

The Lung Cancer Alliance estimates there are over 530 lung cancer screening centers in the U.S. with little attention directed at ensuring educational materials match the literacy level of participants. In general, prior studies have found health related educational materials often exceed the American Medical Association's (AMA) recommended 6th grade reading level making the information incomprehensible to many patients [20-23]. Health literacy is defined as the degree to which individuals have the capacity to obtain, process, and understand health information needed to interpret quantitative information and make appropriate decisions [24, 25]. Only $12 \%$ of Americans have proficient health literacy with low health literacy being more prevalent in the elderly, populations with low SES, the uninsured, and publicly insured patients [24-26].

Understanding and addressing low health literacy in LCS patients is vital to improve outcomes and maintain patient autonomy. In the NLST, $27 \%$ of patients who received the initial screening CT scan were defined as having a positive scan but only $3.8 \%$ of these patients had lung cancer [3]. Providers need to make patients aware that false positive findings are common and up to $25 \%$ of invasive procedures may be performed on cancer-free patients [27]. Patient education is integral to understand this complex process in addition to the possible diagnostic dilemmas that may arise.

Little is known about the literacy level of current educational materials for LCS. The goals of our study were to: 1) assess the health literacy of patients participating in a lung cancer screening program within an urban academic institution, and 2) examine the readability of online educational materials for lung cancer screening.

\section{Methods}

We performed a retrospective cross sectional study at a single urban academic medical center with an established lung cancer screening program. Patients who were eligible for LCS were required to have a shared decision making discussion with their ordering provider (e.g. primary care physicians, pulmonologists, and nurse practitioners) during a clinic visit. However, no standardized decision aids were used during the study period. Only those who agreed to lung cancer screening between June 2015 and June 2017 were included in the study. All patients met criteria for screening based on the United States Preventive Services Task Force recommendations. The most common referral sources were within the center and from affiliated health care centers in the community.

The study protocol was determined to be exempt by the Institutional Review Board (IRB).

\section{Assessment of health literacy}

Participants independently completed a baseline clinic intake form, which included demographic information and a validated three item health literacy screen [28-31]. The response to each health literacy screening question was based on a 5-point Likert scale. The response options for questions one and three were as follows: (1/ never), (2), (3), (4), (5/always). The response options for question two was as follows: (1/unsure), (2), (3), (4), (5/ very sure). The items, shown below, were designed to overcome underreporting and social stigma by asking "how often" rather than "if" participants had a problem [28, 32]. In addition, the literacy screen was validated against the Short Test of Functional Health Literacy in Adults (S-TOFHLA) and the Rapid Estimate of Adult Literacy in Medicine (REALM) to identify patients with inadequate or marginal health literacy [28-31]. This brief screen was selected as opposed to the TOFHLA or REALM to decrease time burden.

Q1. How often do you have problems learning about your medical condition because of difficulty understanding written information? (sensitivity $57 \%$, specificity $78 \%$ ) [28].

Q2. How sure are you filling out medical forms by yourself? (sensitivity $77-80 \%$, specificity $74-77 \%$ ) $[28,30]$.

Q3. How often do you have someone help you read hospital materials? (sensitivity $54-73 \%$, specificity $83 \%$ ) $[28,31]$.

Health literacy was determined by analyzing responses independently as single item screening questions and in combination when completed. Importantly, questions 2 and 3 have been validated in separate studies as single item screening questions to detect low health literacy with comparable sensitivity and specificity $[30,31]$. Question 1, though independently effective, had a lower sensitivity than questions 2 and 3 [28].

The patients were categorized into two groups; inadequate/marginal or adequate health literacy. Similar to prior studies, inadequate health literacy was defined as a reading level $\leq 6$ th grade and marginal health literacy corresponded to a reading level between 7 th and 8th grade [30]. The reading level was assessed based on their response to the validated health literacy screening questions. A cut off of $\geq 3$ for question 1 and question 3 and 
a cutoff of $\leq 3$ for question 2 indicated inadequate/marginal health literacy.

\section{Statistical analysis}

Those who responded to at least one health literacy question (responders) were compared to those who did not complete any literacy screening items (non-responders) to explore if there were demographic differences between the groups. Chi square and t-test analyses were used on categorical and interval data, respectively.

Descriptive statistics were calculated using SPSS Statistics for Mac version 24 (New York, USA). A $p$-value of $\leq 0.05$ was used to determine statistical significance on all analyses.

\section{Assessment of online educational materials}

As a separate analysis, we evaluated the readability level of online educational materials regarding lung cancer screening available to the general population. The Flesch-Kincaid Grade Level (FKGL) readability formula is an objective measure of language complexity [32]. The formula is based on the number of words per sentence and the number of syllables per word. It estimates the U.S. grade level required to understand written material. For example, a FKGL score of 6 indicates the material is written at a 6th grade reading level. The Flesch-Kincaid formula has been used in several studies of readability and demonstrates a high correlation with other readability scales [32]. The formula is as follows:

\subsection{9 (total words/total sentences) +11.8 \\ (total syllables/total words) -15.59}

Using the phrase "What is lung cancer screening?" we performed a Google search on November 17, 2017 for patient focused educational materials from English language websites. Account information and location services were disabled for the search. Web page navigation, copyright notices, postal addresses, phone numbers, uniform resource locaters, disclaimers, date stamps, author information, citations, references, feedback questionnaires, and hyperlinks were not included in the analysis. The first 50 search results were reviewed which included content containing at least 300 words specifically addressing lung cancer screening. The websites contained information on eligibility, imaging tests, cost, risks, and benefits of testing. The FKGL was analyzed using the built in readability tools on Microsoft Word software $[23,33,34]$. The sites were categorized into type of institution providing the content (e.g. academic centers, lung cancer organizations, etc.) and the mean grade level was analyzed per category.

\section{Results}

There were 404 patients who underwent lung cancer screening during the study period, with the majority being referred by primary care physicians $(74 \%)$ followed by pulmonologists (19\%). Demographic characteristics are presented in Table 1. The population was predominantly black $(70.0 \%)$ with $51.5 \%$ being men.

Half of the participants $(n=200,49.5 \%)$ completed at least one health literacy question and were defined as 'responders'. Only 36\% (146/404) answered all three screening questions. Of responders, $44.5 \%(n=89)$ reported an annual income of $\leq \$ 15,000$ and $81 \%(n=162)$ had public insurance. It should be noted non-responders were less likely than responders to reveal their marital status or income, and were more likely to be elderly.

The results of the health literacy questions are presented in Table 2. The prevalence of inadequate/marginal health literacy was $26.7-38.0 \%$, depending on the item(s) used.

\section{Readability of online educational material}

Fifty websites were reviewed and four were excluded from analysis because they were intended for medical providers. The readability level of the 46 websites are presented in Table 3. The mean FKGL for the 46 websites combined was $10.6 \pm 2.2$. Most websites were from academic medical centers (39\%). Only one website, from an academic center, was written at a 6 th grade reading level. In total, ten web sites (21.7\%) were at or below an 8th grade reading level. Government web sites had the lowest mean FKGL $(8.5 \pm 0.96)$ and public websites had the highest $(12.7 \pm 2.63)$.

\section{Discussion}

To our knowledge, this is the first study to consider health literacy in the context of lung cancer screening. Results of literacy screening among our population of inner city, lower income patients revealed approximately one-third of patients had low health literacy. The relevance of this finding is highlighted by the fact the majority of websites identified in a common search that might be conducted by patients (i.e. what is lung cancer screening?) revealed all educational materials, except one, exceeded the AMA recommendations to be written at or below a 6th grade level. The average Flesch-Kincaid Grade Level of online patient educational materials analyzed was 10.6 while the average American reads at an 8th grade level.

The finding that $38 \%$ of patients had low literacy is comparable to results described in similar urban populations who underwent screening for cervical and prostate cancer [35-39]. A pooled analysis of 85 studies found $46 \%$ of patients had low or marginal health literacy which was associated with older age, lower education level, and black patients [40]. Low health 
Table 1 Patient demographics based on responders and non-responders to at least one health literacy question

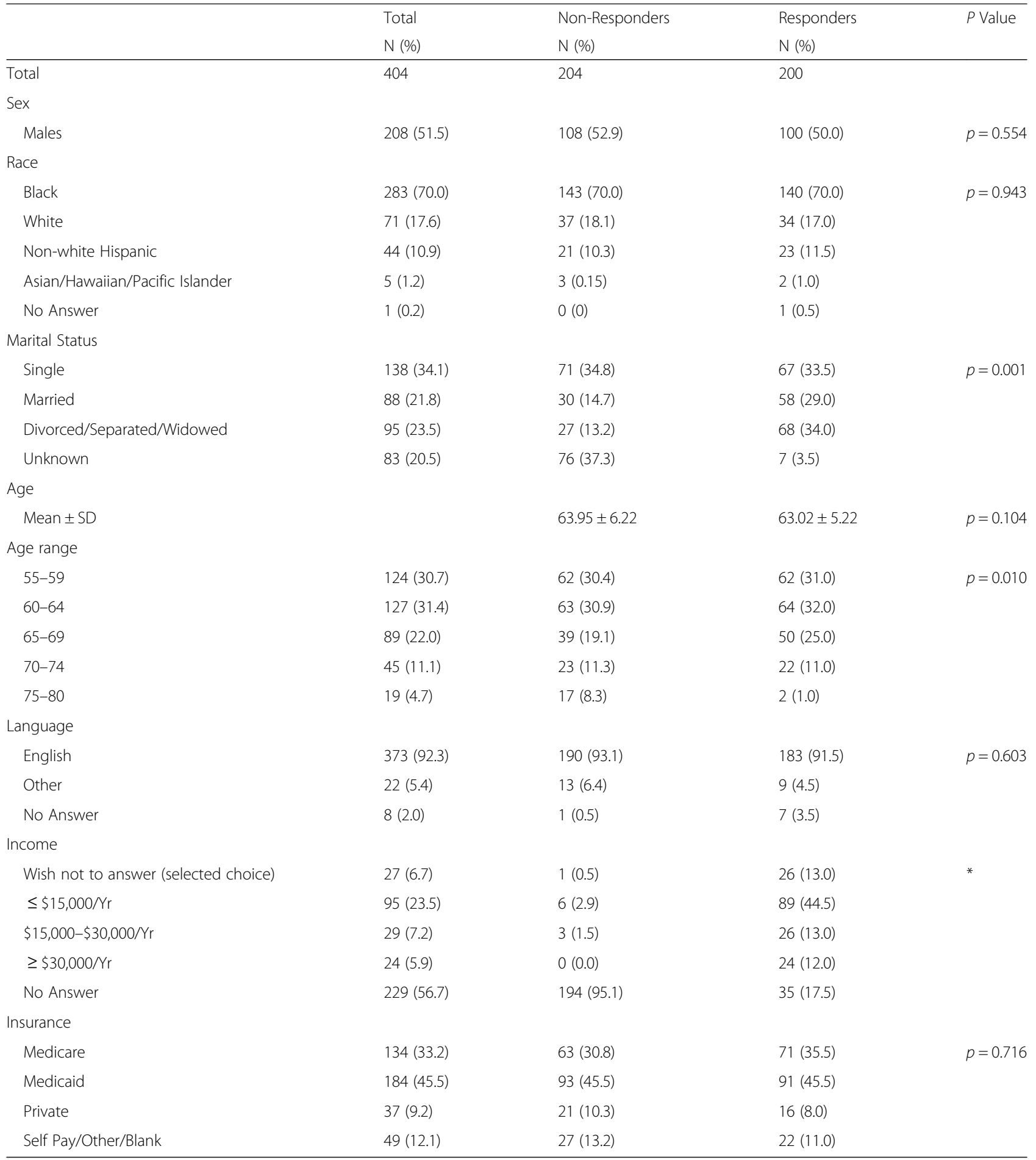

*comparison not performed due to high non-response rate

literacy has been directly linked to worse outcomes across several chronic diseases including, but not limited to, heart disease, diabetes, stroke, advanced kidney disease, asthma, and epilepsy [25, 41-45]. These outcomes may be attributed to limited use of preventative services, higher rates of hospital admissions, increased difficulty managing illnesses, and therefore higher mortality [25, 42, 46, 47].

It is possible the low literacy rate is significantly underestimated in our population. The intake forms were given to patients and they were asked to self-report. It is possible many of the non-responders 
Table 2 Inadequate/marginal health literacy based on responses to specific screening questions

\begin{tabular}{llllll}
\hline & Q1 only & Q2 only & Q3 only & Q2 or Q3 & Q1 or Q2 or Q3 \\
\hline Number of responses & 178 & 173 & 176 & 189 & 200 \\
Number of responses indicating inadequate or marginal literacy (\%) & $30(16.8)$ & $47(27.2)$ & $47(26.7)$ & $65(34.4)$ & $76(38.0)$
\end{tabular}

Q1. How often do you have problems learning about your medical condition because of difficulty understanding written information?(Score $\geq 3$ indicates inadequate/marginal health literacy)

Q2. How sure are you filling out medical forms by yourself?(Score $\leq 3$ indicates inadequate/marginal health literacy)

Q3. How often do you have someone help you read hospital materials? (Score $\geq 3$ indicates inadequate/marginal health literacy)

did not answer the questions due to the stigma of low health literacy and/or due to low health literacy itself. In general, the high rates of low health literacy should raise concerns in a LCS program with a similar population. Recognition of patients with low literacy and optimizing resources to address their educational needs will be critical to the shared decision making process.

In a population similar to our study, lung cancer screening patients preferred information directly from their doctor, and more patients preferred pamphlets/brochures over web-based materials [48]. In the same study, patient knowledge improved with decision aids and 90 min discussion groups. [48]. Another study found improvement in patients' understanding about lung cancer screening with a shared decision making visit using a six-minute video slide show, a decision aid, and a question/answer session [49]. Though patient knowledge did improve in these studies, not all hospitals have the resources to provide this degree of education during a clinic visit. This can cause anxiety, distress, and confusion leading to a significant portion of medical information being forgotten [50-52]. To mitigate these feelings, patients often perform their own medical research on the internet [19]. Unfortunately, we know online information, regardless of disease topic, is written above the literacy level for most patients [20-23]. It has also been shown older patients, patients with lower household incomes, and those with low health literacy are less likely to use the internet for health research, likely due to their difficulty using a computer $[19,53]$. When online material is difficult to comprehend and vulnerable populations have limited access to resources, providing adequate education to those with low health literacy is challenging. Knowledge retention is an additional challenge since this process can take place over several years. Thus, these initial intense interventions may need to be repeated which will be even more resource-consuming.

For patients with low health literacy being referred for LCS to physicians with limited time and resources the best pragmatic option for education is unknown. Our study shows the internet is likely not a viable source. A recent study found similar results indicating most lung cancer screening material is written above the literacy level of the average American [54]. A simpler intervention repeated over time may yield better knowledge transfer and retention in this population. A grade level appropriate pamphlet/decision aid that can be referenced as needed and which simplifies the complexity of lung cancer screening may be a reasonable option. It has been suggested decision aids, though intended for patients, can also improve provider knowledge [13]. When developing educational materials, efforts should be made to keep sentences short, avoid medical jargon, and use simple language associated with pictures. Feedback from target populations is also important when developing educational materials [55].

Our study does have limitations. First, the cohort is from a single urban academic center with a predominantly minority population which limits generalizability. Second, as in any literacy study, there is the potential for non-response bias as patients may feel the questions are too personal or even insulting. Third, the forms used

Table 3 Flesch-Kincaid analysis of lung cancer educational materials

\begin{tabular}{llll}
\hline Source of Web site & N & Grade level range & Average grade level and standard deviation \\
\hline Academic Medical Centers & 18 & $6.4-15.6$ & $10.7 \pm 2.10$ \\
Non-academic Hospital System or Network $^{\mathrm{a}}$ & 8 & $7.1-13.1$ & $10.1 \pm 2.15$ \\
Lung Cancer organizations $^{\text {G }}$ & 5 & $7.4-13.9$ & $10.1 \pm 2.18$ \\
Government $^{b}$ & 3 & $7.5-9.8$ & $8.5 \pm 0.96$ \\
Community/Private Hospitals $_{\text {Public Web sites }}^{c}$ & 9 & $9.0-13.6$ & $11.3 \pm 1.63$ \\
Total $^{c}$ & 3 & $10.1-16.3$ & $12.7 \pm 2.63$ \\
\hline
\end{tabular}

${ }^{a}$ A hospital system is two or more hospitals owned, leased, sponsored, or contract managed by a central organization, while a network is a group of hospitals, physicians, other providers, insurers and/or community agencies that coordinate and deliver a broad spectrum of services to their community [56]

${ }^{\mathrm{b} C e n t e r s}$ for Disease Control and Prevention, United States Preventive Services Task Force, National Cancer Institute

'Wikipedia, Radiologyinfo.org, ShouldIScreen.com 
screening questions rather than more involved measurement tools to determine the literacy rate, however, the questions have been validated. Fourth, patients who were eligible for lung cancer screening but did not receive it were not included in the study, therefore the generalizability for all eligible lung cancer screening patients is limited. Fifth, our health literacy screening questions did not contain word labels for response options 2 , 3 , and 4 on the Likert scale. All the referenced screening questions associated option 3 , the middle option, with the word 'sometimes.' Therefore, we cannot assume our unlabeled option 3 is equivalent to the label 'sometimes' noted in the references. Finally, the focus on English language websites and inclusion of predominately English speaking patients also limits generalizability.

\section{Conclusion}

In our population, low health literacy was common and is likely a barrier to appropriate education for lung cancer screening. The current online educational materials regarding lung cancer screening are written above the recommended reading level set by the AMA, which may further increase patient confusion and anxiety. As more physicians order lung cancer screening for their patients, the need for educational materials directed at those with low health literacy needs to be further explored. Optimizing resources to address these educational needs will be a challenge but is essential to improve the lung cancer screening process.

\begin{abstract}
Abbreviations
AMA: American Medical Association; FKGL: Flesch-Kincaid Grade Level; LCS: Lung cancer screening; NLST: National lung cancer screening trial; PCP: Primary care physicians; REALM: Rapid estimate of adult literacy in medicine; SES: Socioeconomic status; TOFHLA: Test of functional health literacy in adults
\end{abstract}

\section{Acknowledgements}

Not applicable.

\section{Funding}

This research was supported by the Respiratory Health Association. The content is solely the responsibility of the authors and does not necessarily represent the official views of the Respiratory Health Association. The Respiratory Health Association did not have a role in the design, collection, analysis, and interpretation of data and writing the manuscript.

\section{Availability of data and materials}

The datasets used and/or analyzed during the current study are available from the corresponding author on reasonable request.

\section{Authors' contributions}

$\mathrm{KH}$ had full access to the data and takes full responsibility for the integrity of the data, accuracy of the data analysis, and integrity of the study as a whole, from inception to publication. KH, CB, LS, AK, MP, LF, KK, MJ made substantial contributions to the conception and design, analysis, and interpretation of data, drafting and revising of manuscript, and read and approved the final manuscript. KH, CB, LS, AK, MP, LF, KK, MJ agree to be accountable for all aspects of the work.
Ethics approval and consent to participate

Study was approved by the Institutional Review Board (IRB) of the University of Illinois as an exempted study. The need for informed consent was waived by the IRB based on the Unites States Department of Health and Human

Services code 46.116 for requirements for informed consent.

Consent for publication

Not applicable.

\section{Competing interests}

The authors declare that they have no competing interests.

\section{Publisher's Note}

Springer Nature remains neutral with regard to jurisdictional claims in published maps and institutional affiliations.

\section{Author details}

${ }^{1}$ University of Illinois at Chicago, 840 S. Wood St., CSB 915, MC 719, Chicago, IL 60612, USA. ${ }^{2}$ University of Illinois College of Pharmacy, 463 Westside Research Office Bldg. 1747 West Roosevelt Road, Chicago, IL 60608, USA. ${ }^{3}$ University of Illinois at Chicago, 840 S Wood Street, 820-E CSB, MC 713, Chicago, IL 60612, USA.

Received: 14 May 2018 Accepted: 28 November 2018

Published online: 07 December 2018

\section{References}

1. Alberg AJ, Brock MV, Ford JG, Samet JM, Spivack SD. Epidemiology of lung Cancer: diagnosis and Management of Lung Cancer, 3rd ed: American College of Chest Physicians Evidence-Based Clinical Practice Guidelines. Chest. 2013;143(5 Suppl):e1S-e29S.

2. Tanner NT, Gebregziabher M, Hughes Halbert C, Payne E, Egede LE, Silvestri GA. Racial differences in outcomes within the National Lung Screening Trial. Implications for widespread implementation. Am J Respir Crit Care Med. 2015:192(2):200-8.

3. National Lung Screening Trial Research, Aberle DR T, Adams AM, Berg CD, Black WC, Clapp JD, Fagerstrom RM, Gareen IF, Gatsonis C, Marcus PM, et al. Reduced lung-cancer mortality with low-dose computed tomographic screening. N Engl J Med. 2011;365(5):395-409.

4. Cancer Stat Facts: Lung and Bronchus Cancer [https://seer.cancer.gov/ statfacts/html/lungb.html].

5. Goldstraw P, Crowley J, Chansky K, Giroux DJ, Groome PA, Rami-Porta R, Postmus PE, Rusch V, Sobin L, International Association for the Study of Lung Cancer international staging C, et al. The IASLC lung Cancer staging project: proposals for the revision of the TNM stage groupings in the forthcoming (seventh) edition of the TNM classification of malignant tumours. J Thorac Oncol. 2007;2(8):706-14.

6. Pinsky PF, Ford M, Gamito E, Higgins D, Jenkins V, Lamerato L, Tenorio S, Marcus PM, Gohagan JK. Enrollment of racial and ethnic minorities in the prostate, lung, colorectal and ovarian Cancer screening trial. J Natl Med Assoc. 2008;100(3):291-8

7. Gross CP, Filardo G, Mayne ST, Krumholz HM. The impact of socioeconomic status and race on trial participation for older women with breast cancer. Cancer. 2005;103(3):483-91.

8. Hussain-Gambles M, Atkin K, Leese B. Why ethnic minority groups are under-represented in clinical trials: a review of the literature. Health Soc Care Community. 2004;12(5):382-8.

9. Hamel LM, Penner LA, Albrecht TL, Heath E, Gwede CK, Eggly S. Barriers to clinical trial enrollment in racial and ethnic minority patients with Cancer. Cancer Control. 2016;23(4):327-37.

10. Al-Refaie WB, Vickers SM, Zhong W, Parsons H, Rothenberger D, Habermann EB. Cancer trials versus the real world in the United States. Ann Surg. 2011; 254(3):438-42 discussion 442-433.

11. Du W, Gadgeel SM, Simon MS. Predictors of enrollment in lung cancer clinical trials. Cancer. 2006;106(2):420-5.

12. Cancer Health Disparities - National Cancer Institute [https://www.cancer. gov/about-nci/organization/crchd/cancer-health-disparities-fact-sheet]

13. Triplette M, Kross EK, Mann BA, Elmore JG, Slatore CG, Shahrir S, Romine PE, Frederick PD, Crothers K. An assessment of primary care and pulmonary provider perspectives on lung Cancer screening. Ann Am Thorac Soc. 2018; 15(1):69-75. 
14. Mazzone PJ, Silvestri GA, Patel S, Kanne JP, Kinsinger LS, Wiener RS, Soo Hoo G, Detterbeck FC. Screening for lung Cancer: CHEST guideline and expert panel report. Chest. 2018;153(4):954-85.

15. Kanodra NM, Pope C, Halbert CH, Silvestri GA, Rice $\amalg$, Tanner NT. Primary care provider and patient perspectives on lung Cancer screening. A qualitative study. Ann Am Thorac Soc. 2016;13(11):1977-82.

16. Henderson LM, Jones LM, Marsh MW, Brenner AT, Goldstein AO, Benefield TS, Greenwood-Hickman MA, Molina PL, Rivera MP, Reuland DS. Opinions, practice patterns, and perceived barriers to lung cancer screening among attending and resident primary care physicians. Risk Manag Healthc Policy. 2017;10:189-95.

17. Legare F, Ratte S, Gravel K, Graham ID. Barriers and facilitators to implementing shared decision-making in clinical practice: update of a systematic review of health professionals' perceptions. Patient Educ Couns. 2008;73(3):526-35

18. Gravel K, Legare F, Graham ID. Barriers and facilitators to implementing shared decision-making in clinical practice: a systematic review of health professionals' perceptions. Implement Sci. 2006;1:16.

19. Diaz JA, Griffith RA, Ng JJ, Reinert SE, Friedmann PD, Moulton AW. Patients' use of the internet for medical information. J Gen Intern Med. 2002;17(3): 180-5.

20. Weiss BD: Health literacy and patient safety: Help patients understand. $\neg$ †Manual for clinicians, Second edn: American Medical Association Foundation and American Medical Association; 2009.

21. Hansberry DR, John A, John E, Agarwal N, Gonzales SF, Baker SR. A critical review of the readability of online patient education resources from RadiologyInfo.Org. AJR Am J Roentgenol. 2014;202(3):566-75.

22. Roberts H, Zhang D, Dyer GS. The readability of AAOS patient education materials: evaluating the Progress since 2008. The Journal of bone and joint surgery American. 2016;98(17):e70.

23. Badarudeen $S$, Sabharwal S. Assessing readability of patient education materials: current role in Orthopaedics. Clin Orthop Relat Res. 2010;468(10): 2572-80.

24. Kutner M, Greenberg E, Jin Y, Paulsen C, White S. The health literacy of America's adults: results from the 2003 National Assessment of adult literacy. Washington, DC: National Center for education Statistics; 2006.

25. Berkman ND, Sheridan SL, Donahue KE, Halpern DJ, Crotty K. Low health literacy and health outcomes: an updated systematic review. Ann Intern Med. 2011;155(2):97-107.

26. Paasche-Orlow MK, Parker RM, Gazmararian JA, Nielsen-Bohlman LT, Rudd RR. The prevalence of limited health literacy. J Gen Intern Med. 2005;20(2):175-84.

27. Detterbeck FC, Mazzone PJ, Naidich DP, Bach PB. Screening for lung cancer: diagnosis and management of lung cancer, 3rd ed: American College of Chest Physicians evidence-based clinical practice guidelines. Chest. 2013; 143(5 Suppl):e78S-92S

28. Chew LD, Bradley KA, Boyko EJ. Brief questions to identify patients with inadequate health literacy. Fam Med. 2004;36(8):588-94.

29. Chew LD, Griffin JM, Partin MR, Noorbaloochi S, Grill JP, Snyder A, Bradley KA, Nugent SM, Baines AD, VanRyn M. Validation of screening questions for limited health literacy in a large VA outpatient population. J Gen Intern Med. 2008;23(5):561-6.

30. Wallace LS, Rogers ES, Roskos SE, Holiday DB, Weiss BD. Brief report: screening items to identify patients with limited health literacy skills. J Gen Intern Med. 2006;21(8):874-7.

31. Morris NS, MacLean CD, Chew LD, Littenberg B: The single item literacy screener: evaluation of a brief instrument to identify limited reading ability. In: BMC Fam Pract. Volume 7, edn; 2006: 21.

32. Kincaid J, RP F, Rogers R, Chissom B. Derivation of new readability formulas (Automated Readability Index, Fog Count, and Flesch Reading Ease Formula) for Navy enlisted Personnel Research Branch report 8-75. Memphis: Naval Air Station; 1975.

33. Ley P, Florio T. The use of readability formulas in health care; 2007. https:// doi.org/10.1080/13548509608400003.

34. Hoffman-Goetz L, Friedman DB. A systematic review of readability and comprehension instruments used for print and web-based Cancer information. Health Educ Behav. 2006;33.

35. Sharp LK, Zurawski JM, Roland PY, O'Toole C, Hines J. Health literacy, cervical cancer risk factors, and distress in low-income African-American women seeking colposcopy. Ethnicity \& disease. 2002;12(4):541-6.

36. Bennett $\mathrm{CL}$, Ferreira MR, Davis TC, Kaplan J, Weinberger M, Kuzel T, Seday MA, Sartor O. Relation between literacy, race, and stage of presentation among low-income patients with prostate cancer. Journal of clinical oncology : official journal of the American Society of Clinical Oncology. 1998;16(9):3101-4.

37. Kim SP, Knight SJ, Tomori C, Colella KM, Schoor RA, Shih L, Kuzel TM, Nadler RB, Bennett CL. Health literacy and shared decision making for prostate cancer patients with low socioeconomic status. Cancer Investig. 2001;19(7): 684-91.

38. Lindau ST, Tomori C, Lyons T, Langseth L, Bennett CL, Garcia P. The association of health literacy with cervical cancer prevention knowledge and health behaviors in a multiethnic cohort of women. Am J Obstet Gynecol. 2002;186(5):938-43.

39. Zahnd WE, Scaife SL, Francis ML. Health literacy skills in rural and urban populations. Am J Health Behav. 2009;33(5).

40. Paasche-Orlow MK. Department of Medicine BUSoM, Boston, MA, USA, Parker RM, Department of Medicine EUSoM, Atlanta, GA, USA, Gazmararian JA, Department of Health Policy and Management RSOPH, Emory University, Atlanta, GA, USA, Nielsen-Bohlman LT, board on neuroscience and behavioral health loM, Washington, DC, USA, Rudd RR, Department of Society HD, and health, Harvard School of Public Health, Boston, MA, USA: the prevalence of limited health literacy. J Gen Intern Med. 2005;20(2):17584.

41. DeWalt DA, Malone RM, Bryant ME, Kosnar MC, Corr KE, Rothman RL, Sueta CA, Pignone MP. A heart failure self-management program for patients of all literacy levels: a randomized controlled trial. BMC Health Serv Res. 2006;6: 30

42. Bostock S, Steptoe A. Association between low functional health literacy and mortality in older adults: longitudinal cohort study. BMJ. 2012.

43. Cavanaugh KL, Wingard RL, Hakim RM, Eden S, Shintani A, Wallston KA, Huizinga MM, Elasy TA, Rothman RL, Ikizler TA. Low health literacy associates with increased mortality in ESRD. Journal of the American Society of Nephrology : JASN. 2010;21(11):1979-85.

44. Taylor DM, Bradley JA, Bradley C, Draper H, Johnson R, Metcalfe W, Oniscu G, Robb M, Tomson C, Watson C, et al. Limited health literacy in advanced kidney disease. Kidney Int. 2016;90(3):685-95.

45. Brigo F, Otte WM, Igwe SC, Tezzon F, Nardone R. Clearly written, easily comprehended? The readability of websites providing information on epilepsy. Epilepsy \& behavior : E\&B. 2015;44:35-9.

46. Rowlands G, Protheroe J, Winkley J, Richardson M, Seed PT, Rudd R. A mismatch between population health literacy and the complexity of health information: an observational study. The British journal of general practice : the journal of the Royal College of General Practitioners. 2015;65(635):e37986.

47. Sudore RL, Yaffe K, Satterfield S, Harris TB, Mehta KM, Simonsick EM, Newman AB, Rosano C, Rooks R, Rubin SM, et al. Limited literacy and mortality in the elderly: the health, aging, and body composition study. J Gen Intern Med. 2006;21(8):806-12.

48. Crothers K, Kross EK, Reisch LM, Shahrir S, Slatore C, Zeliadt SB, Triplette M, Meza R, Elmore JG. Patients' Attitudes Regarding Lung Cancer Screening and Decision Aids. A Survey and Focus Group Study; 2016. https://www. atsjournals.org/doi/10.1513/AnnalsATS.201604-2890C.

49. Mazzone PJ, Tenenbaum A, Seeley M, Petersen H, Lyon C, Han X, Wang XF. Impact of a lung Cancer screening counseling and shared decision-making visit. Chest. 2017;151(3):572-8.

50. Gressard L, DeGroff AS, Richards TB, Melillo S, Kish-Doto J, Heminger CL, Rohan EA, Allen KG: A qualitative analysis of smokers' perceptions about lung cancer screening. In: BMC Public Health. Volume 17, edn.; 2017.

51. Slatore CG, Wiener RS. Pulmonary nodules: a small problem for many, severe distress for some, and how to communicate about it. Chest. 2017.

52. Kessels RP. Patients' memory for medical information. J R Soc Med. 2003; 96(5):219-22.

53. Bailey SC, O'Conor R, Bojarski EA, Mullen R, Patzer RE, Vicencio D, Jacobson KL, Parker RM, Wolf MS. Literacy disparities in patient access and healthrelated use of internet and mobile technologies. Health Expect. 2015;18(6): 3079-87.

54. Hansberry DR, White MD, D'Angelo M, Prabhu AV, Kamel S, Lakhani P, Sundaram B. Lung Cancer screening guidelines: how readable are internetbased patient education resources? AJR Am J Roentgenol. 2018;211(1):W42-6.

55. Wong LP. Focus group discussion: a tool for health and medical research. Singap Med J. 2008;49(3):256-60 quiz 261.

56. AHA Hospital Statistics: Fast Facts on US Hospitals In: 2017 